\title{
An inventory of national surveillance systems assessing physical activity, sedentary behaviour and sport participation of adults in the European Union
}

Maroje Sorić ${ }^{12^{*}}$, Kaja Meh', Paulo Rocha ${ }^{3}$, Wanda Wendel-Vos ${ }^{4}$, Ellen de Hollander ${ }^{4}$ and Gregor Jurak ${ }^{1}$

\begin{abstract}
Background: Physical inactivity has been recognised as a global public health problem that requires concerted action. This calls for systematic physical activity (PA) surveillance as a mechanism for assessing the problem and evaluating the effectiveness of related policies. Because countries tend to design their policy measures based on national surveillance data, here we present an inventory of existing national surveillance systems on PA, sedentary behaviour (SB) and sport participation (SP) among adult population in all European Union (EU) Member States.

Methods: As a part of the European Physical Activity and Sports Monitoring System (EUPASMOS) project, a questionnaire was constructed in the form of an on-line survey to collect detailed information on existing national surveillance systems on either PA, SB, or SP. National HEPA focal points from all 27 EU Member States were invited to answer the on-line questionnaire and data collection took part in the period May 2018-September 2019.

Results: National monitoring of PA or SB or SP for adults has been established in 16/27 EU Member States, that host 33 different PA/SB/SP monitoring systems. Apart from 3 countries that are using accelerometers (Finland, Ireland and Portugal), surveillance is typically based on questionnaires. In most Member States these questionnaires have not been validated in the particular language and cultural setting. Next, specific domains and dimensions of PA, SB and SP assessed vary a lot across countries. Only 3 countries (the Netherlands, Portugal and Slovenia) are monitoring all three behaviours while covering most of the domains and dimensions of PA/SB/SP. Lastly, as half of the existing surveillance systems set an upper age limit, in 9/16 countries that are monitoring PA/SB/SP, no data for people older than 80 years are available.
\end{abstract}

\footnotetext{
* Correspondence: maroje.soric@fsp.uni-lj.si

${ }^{1}$ Faculty of Sport, University of Ljubljana, Gortanova 22, 1000 Ljubljana,

Slovenia

${ }^{2}$ Faculty of Kinesiology, University of Zagreb, Zagreb, Croatia

Full list of author information is available at the end of the article
}

C The Author(s). 2021 Open Access This article is licensed under a Creative Commons Attribution 4.0 International License, which permits use, sharing, adaptation, distribution and reproduction in any medium or format, as long as you give appropriate credit to the original author(s) and the source, provide a link to the Creative Commons licence, and indicate if changes were made. The images or other third party material in this article are included in the article's Creative Commons licence, unless indicated otherwise in a credit line to the material. If material is not included in the article's Creative Commons licence and your intended use is not permitted by statutory regulation or exceeds the permitted use, you will need to obtain permission directly from the copyright holder. To view a copy of this licence, visit http://creativecommons.org/licenses/by/4.0/. The Creative Commons Public Domain Dedication waiver (http://creativecommons.org/publicdomain/zero/1.0/) applies to the data made available in this article, unless otherwise stated in a credit line to the data. 
Conclusions: Systematic surveillance of PA is lacking among 11/27 EU countries, with even few monitoring SB and SP. Besides, existing surveillance systems typically fail to assess all dimensions and domains of PA/SB/SP with only three countries maintaining monitoring systems that encompass all three behaviours while covering most of the domains and dimensions of PA/SB/SP. Hence, additional efforts in advocacy of systematic PA surveillance in the EU are called for.

Keywords: 24-h movement behaviour, Physical activity questionnaire, Physical inactivity, Sitting, Sedentarism, Sedentary behaviour questionnaire, Healthy lifestyle, Physical activity measurement

\section{Background}

Owing to its strong associations with a range of chronic diseases and premature mortality, both low physical activity (PA) and high sedentary behaviour (SB) are widely recognised as a global public health problem that requires concerted action [1]. Furthermore, physical inactivity does not only affect individual health, but also burdens national economies with increasing health care costs and productivity losses. The most recent estimates reveal that direct health care costs of physical inactivity in Europe reach international $\$ 11.7$ billion per year, in addition to $\$ 3.8$ billion of productivity losses [2]. To address the physical inactivity pandemics, a series of strategic documents were adopted on both global and European level [3-9]. All these documents call for PA and physical fitness surveillance systems as a means for monitoring trends and evaluating the effectiveness of the action plans. Additionally, a need for a harmonised public health surveillance system of the European population has been identified to obtain comparable data across countries and align their policies and action plans [10]. With strong political and legislative support [11] focusing on adults, the European Union (EU) established the European Health Interview Survey (EHIS) coordinated by Eurostat and run by national statistical offices to meet this challenge [12]. Within EHIS also data on $\mathrm{PA}$ and $\mathrm{SB}$ are being collected with a sub-module through a short PA questionnaire (EHIS-PAQ). The EHIS-PAQ [13] consists of 8 items covering PA during work, transportation, and leisure time as well as musclestrengthening exercise during a typical week. Still, it does not provide much detail on intensity of PA, and has been shown to have only moderate validity [14]. Moreover, EHIS survey relies on output harmonisation and does not mandate a consistent questionnaire across all Member States, resulting in non-equivalent and sometimes missing information for some aspects of PA or SB. Therefore, a comprehensive picture of population trends and regional variations of the levels of PA and sedentariness in the EU can be obtained only by combining information from cross-national European surveillance systems and national ones, especially in countries that monitor PA with more valid tools (e.g., accelerometers), as well as with information from countries that monitor sport participation (SP) as a very important domain of PA. This combination of sources would enable more precise identification of PA characteristics across regions and over time, as well as a better evaluation of policy actions.

Two large overviews on surveillance systems which include population-based measures of PA performed by the World Health Organisation (WHO) in 2015 [15] and 2018 [16] concluded that the vast majority of the EU Member States are monitoring PA. Yet, no effort was made to distinguish between international and national systems, nor was a systematic nature of data collection and usage required in the definition of surveillance. In addition, the Determinants of diet and physical activity study (DEDIPAC), which gathered information on international, European regional and national surveillance systems on diet, PA and SB in 11 countries, identified three multinational European surveys, and a number of national and regional surveys on PA and SB among adult population [17]. However, although this study offered a comprehensive overview of EU-wide surveillance systems, it provided data on national surveillance for only selected few EU Member States.

Hence, there is a need for a thorough inventory of national surveillance systems on PA and SB across the EU. Additionally, data on SP could enrich this overview since sport activities represent an important domain of PA and exhibit large positive effects not just on physical, but also on mental and social health. To bridge this gap, an inventory on existing national surveillance systems on PA, SB and SP for adults in all EU Member States was prepared within the European Physical Activity and Sports Monitoring System (EUPASMOS) project. This paper summarizes and discusses its main outcomes, contributing to the development of the framework for national surveillance systems in these areas.

\section{Methods}

A web-based questionnaire was constructed to seek data on existing national surveillance systems on either: 1) $\mathrm{PA}$; 2) SB; or 3) SP. The questionnaire was constructed specifically for the purpose of this study and consisted of 48 items. For each country data on number of different monitoring systems was collected as well as details of 
each monitoring system (e.g., years of monitoring, age span of participants, number of participants). In the next section, data on domains and dimensions of PA, SB and $\mathrm{SP}$ measured in each national monitoring system were collected through multiple-choice questions. The last section of the questionnaire inquired which of the PA questionnaires used had been formally validated and gathered information about the use of device-based assessment in surveillance of PA.

Prior to data collection, the questionnaire was pilottested among EUPASMOS members. Then, in May 2018, national focal points of the European network for the promotion of health-enhancing physical activity (HEPA Europe) from all 27 EU Member States were invited to answer the on-line questionnaire. HEPA Europe is a WHO/Europe network, established in 2005 with the mission to provide a forum for the advancement of HEPA research, policy and practice across the WHO European Region. In 2013, EU Member States have been requested by the European Commission to appoint national physical activity focal points to support the framework to monitor HEPA policies. After having resolved issues on missing or incomplete information, data gathering was closed in September 2019, with 20 Member States having filled out the online questionnaire. In total, 18 countries provided all the requested information, two countries offered partial information, and seven countries didn't complete the online questionnaire at all. After that, we collected data for the remaining seven Member States and for two Member States which filled out the online questionnaire, but some data were incomplete (i.e., Austria, Belgium, Denmark, Finland, Germany, Greece, Italy, Slovakia and Spain) through the information available in the DEDIPAC study [17] and the WHO's PA country factsheets [16]. Additionally, we consulted national experts from all mentioned Member States to get information equivalent to the inventory questionnaire.

All methods and procedures of this study were in accordance with the Declaration of Helsinki and were approved by the Ethics Committee of the Faculty of Sport at the University of Ljubljana (ID: 10/2018). Having fully informed the participants about the aims and procedures of the study, their verbal consent was obtained. As only administrative data were recorded, and no personal data were collected, written informed consent was not required.

\section{Eligibility criteria}

First, for the purpose of this inventory a surveillance system was defined as a systematic collection, analysis and interpretation of the health-related data needed for the planning, implementation, and evaluation of public health practice. Only systems that regularly monitor population level of physical activity, sedentary behaviours or sport participation in adults (18+ years), regardless of the sector from which the system stems (e.g., health, education, industry etc.), were considered eligible. Single cross-sectional, cohort and intervention studies that contain data on $\mathrm{PA} / \mathrm{SB} / \mathrm{SP}$ were excluded. In addition, as countries tend to design their policy measures based on national surveillance data, only national monitoring systems, and not local, regional or panEuropean ones (e.g., EHIS, Eurobarometer), were considered eligible for inclusion in this inventory.

\section{Results}

Figure 1 shows the availability of national $\mathrm{PA} / \mathrm{SB} / \mathrm{SP}$ monitoring systems across the EU. At the time of this study, national monitoring of PA, SB or SP for adults had been set up in 16/27 EU Member States (Belgium, Denmark, Estonia, Finland, Germany, Greece, Ireland, Italy, Latvia, Lithuania, the Netherlands, Portugal, Romania, Slovenia, Spain and Sweden). Sixteen Member States with established monitoring systems host 33 different $\mathrm{PA} / \mathrm{SB} / \mathrm{SP}$ surveillance systems. Nearly all these systems $(N=28)$ assess $\mathrm{PA}$, while $\mathrm{SP}$ is evaluated in 15 national systems, and SB in 11.

Only 8 EU Member States are monitoring all three behaviours on a national level (Denmark, Finland, Ireland, the Netherlands, Portugal, Romania, Slovenia and Spain). In Belgium, Estonia, Germany, Greece and Italy, SB is not being monitored, while Lithuania and Sweden are not monitoring SP. Most national monitoring systems combine PA and SP $(N=10)$, or PA and SB $(N=8)$ in one survey. Twelve systems focus on only one of these behaviours (8 PA and $4 \mathrm{SP}$ ), while the Netherlands, Portugal and Romania assess all 3 behaviours in a single system.

A list of the monitoring systems and their general characteristics is provided in Table 1 , while Table 2 summarises constructs, domains and dimensions covered, as well as the methods of assessment employed in each individual survey.

National PA/SB/SP monitoring systems in the EU are equally often based on interviews and self-administered questionnaires. Two predominant modes of administration are pen and paper $(N=16)$ and computer or online surveys $(N=14)$, while other means are used less frequently: telephone interviews $(N=10)$, and face to face interviews $(N=8)$.

The age range of participants across national $\mathrm{PA} / \mathrm{SB} /$ SP monitoring systems differs to a great extent. Generally, systems include participants starting from the age of 15-18, while two systems are measuring $\mathrm{PA} / \mathrm{SB} / \mathrm{SP}$ exclusively in elderly (Finland and Ireland). On the other side, most systems have set the upper age limit for participation. Hence, in 9/16 countries that monitor PA/SB/ 


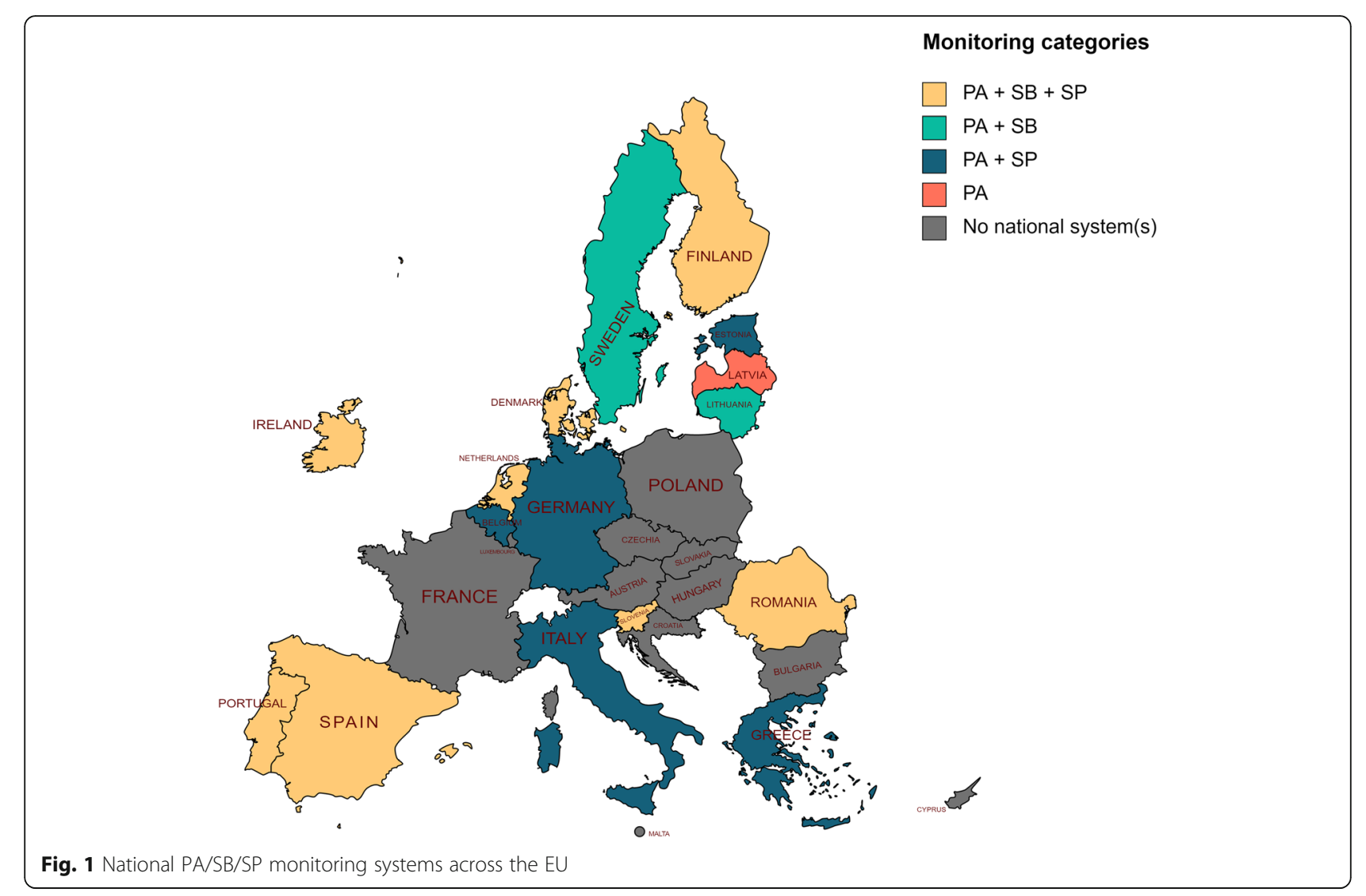

SP no data for people older than 80 years are being collected.

Only seven countries are monitoring at least one of the behaviours annually (Estonia, Finland, Greece, Ireland, Italy, Lithuania, the Netherlands). In five countries PA, SB or SP are being monitored every 2-3 years (Denmark, Latvia, Romania, Slovakia and Sweden), while in Belgium, Portugal and Spain this is done less than every 3 years. Finally, Germany and Slovenia have reported irregular time intervals for monitoring PA/SB/SP.

Most of the national PA monitoring systems are embedded in larger surveys $(N=27)$, while Denmark, Estonia, Ireland, Lithuania and Spain run systems that focus exclusively on PA/SB/SP.

Figure 2 shows the number of systems and countries monitoring specific domains and dimensions of PA, SB and SP. It seems that PA and SB are most frequently monitored during leisure time, while the domains of SP are typically not specified. Frequency and duration are the most often reported dimensions of PA and SP, whereas sitting is the most commonly included dimension of SB. It should be noted that only the Netherlands, Portugal and Slovenia are running monitoring systems that encompass all three behaviours, while covering most of the domains and dimensions of PA/SB/SP.
Methods for assessing PA/SB/SP used in the identified monitoring systems are far from uniform. A lot of national PA systems are using custom questionnaires, developed specifically for their surveillance system (17 systems across 11 countries). Conversely, some countries rely on popular international questionnaires within their monitoring systems; most often on the International Physical Activity Questionnaire (IPAQ; used in 10 systems across 8 countries; mostly in short form). Most of the questionnaires being used needed to be crossculturally adapted, but their measurement properties were not examined after the process. In effect, only six countries are using validated interpretations of original or adapted questionnaires in their national monitoring systems: Ireland, Portugal, Spain and Sweden had validated the IPAQ - short form, Denmark had validated the Marshall D questionnaire and the Netherlands had validated the SQUASH. Finally, only three countries (Finland, Ireland and Portugal) include device-measured PA (i.e., accelerometery) in their monitoring systems.

\section{Discussion}

We gathered data about national systems that monitor $\mathrm{PA} / \mathrm{SB} / \mathrm{SP}$ of the adult population from all 27 countries in the EU. We identified different systems, but only analysed national, and not regional systems, and only 
Table 1 Basic characteristics of movement behaviours monitoring systems across the European Union

\begin{tabular}{|c|c|c|c|c|c|c|c|}
\hline \multirow{2}{*}{$\begin{array}{l}\text { Country, name of the } \\
\text { system and } \\
\text { (abbreviation) }\end{array}$} & \multirow[t]{2}{*}{ Years of monitoring } & \multirow{2}{*}{$\begin{array}{l}\text { Frequency } \\
\text { of } \\
\text { monitoring }\end{array}$} & \multirow{2}{*}{$\begin{array}{l}\text { App. } \\
\text { number of } \\
\text { participants }\end{array}$} & \multirow{2}{*}{$\begin{array}{l}\text { Age range } \\
\text { of } \\
\text { participants }\end{array}$} & \multicolumn{2}{|c|}{ Administration } & \multirow{2}{*}{$\begin{array}{l}\text { Embedded } \\
\text { or } \\
\text { exclusively } \\
\text { PA/SB/SP }\end{array}$} \\
\hline & & & & & Mode & Means & \\
\hline \multicolumn{8}{|l|}{ Belgium $^{\mathrm{a}}$} \\
\hline $\begin{array}{l}\text { Belgian National Food } \\
\text { Consumption Survey } \\
\text { (BNFCS) }\end{array}$ & 2004,2014 & 10 years & 1200 & $18-64$ & $\begin{array}{l}\text { Interview, } \\
\text { Self- } \\
\text { administered }\end{array}$ & $\begin{array}{l}\text { Face to face interview, } \\
\text { Computer-assisted }\end{array}$ & Embedded \\
\hline BHIS & $\begin{array}{l}\text { 1997, 2001, 2008, 2013, } \\
2018\end{array}$ & 5 years & 10,000 & $19-64$ & $\begin{array}{l}\text { Interview, } \\
\text { Self- } \\
\text { administered }\end{array}$ & $\begin{array}{l}\text { Face to face interview, } \\
\text { Computer aided } \\
\text { personal interview }\end{array}$ & Embedded \\
\hline \multicolumn{8}{|l|}{ Denmark $^{a}$} \\
\hline $\begin{array}{l}\text { Den Nationale } \\
\text { Sundhedsprofil (DNS) }\end{array}$ & 2010, 2013, 2017 & $3-4$ years & 520,000 & $16+$ & $\begin{array}{l}\text { Self- } \\
\text { administered }\end{array}$ & $\begin{array}{l}\text { Pen and paper, } \\
\text { computer-assisted }\end{array}$ & $\begin{array}{l}\text { Exclusively } \\
\mathrm{PA} / \mathrm{SB} / \mathrm{SP}\end{array}$ \\
\hline $\begin{array}{l}\text { Danskernes motions- } \\
\text { og sportsvaner (DMS) }\end{array}$ & $\begin{array}{l}\text { 1964, 1975, 1987, 1993, } \\
\text { 1998, 2002, 2004, 2007, } \\
2011,2016\end{array}$ & Random & 33,000 & $18+$ & $\begin{array}{l}\text { Interview, } \\
\text { Self- } \\
\text { administered }\end{array}$ & $\begin{array}{l}\text { Face to face interview, } \\
\text { Telephone interview, } \\
\text { computer-assisted c }\end{array}$ & $\begin{array}{l}\text { Exclusively } \\
\mathrm{PA} / \mathrm{SB} / \mathrm{SP}\end{array}$ \\
\hline \multicolumn{8}{|l|}{ Estonia } \\
\hline $\begin{array}{l}\text { Health Behaviour } \\
\text { among Estonian } \\
\text { Adult Population } \\
\text { (HBEAP) }\end{array}$ & 1993-2017 & 2 years & 5500 & $18-74$ & Interview & Pen and paper & Embedded \\
\hline $\begin{array}{l}\text { National Physical } \\
\text { Activity Survey } \\
\text { among Estonian } \\
\text { Adult Population } \\
\text { (NPASEAP) }\end{array}$ & $2015-2018$ & Annual & 1000 & $15-69$ & Interview & $\begin{array}{l}\text { Telephone aided } \\
\text { personal interview }\end{array}$ & $\begin{array}{l}\text { Exclusively } \\
\text { PA/SB/SP }\end{array}$ \\
\hline \multicolumn{8}{|l|}{ Finland $^{\mathrm{a}}$} \\
\hline $\begin{array}{l}\text { National FinHealth } \\
\text { Study (FinHealth) }\end{array}$ & 2017 to date & 5 years & 7050 & $18+$ & $\begin{array}{l}\text { Self- } \\
\text { administered, } \\
\text { Interview }\end{array}$ & $\begin{array}{l}\text { Computer or online } \\
\text { survey, Telephone } \\
\text { interview }\end{array}$ & Embedded \\
\hline $\begin{array}{l}\text { Terveys } 2011 \text { (Health } \\
\text { 2000-2011) }\end{array}$ & $2000-2011$ & 10 years & 9000 & $18+$ & $\begin{array}{l}\text { Self- } \\
\text { administered, } \\
\text { Interview }\end{array}$ & $\begin{array}{l}\text { Pen and paper, } \\
\text { Telephone interview }\end{array}$ & Embedded \\
\hline $\begin{array}{l}\text { Health Behaviour and } \\
\text { Health among the } \\
\text { Finnish Retirement- } \\
\text { Age Population (EVTK) }\end{array}$ & 1985-2013 & Annual & 2400 & $65-84$ & $\begin{array}{l}\text { Self- } \\
\text { administered }\end{array}$ & Pen and paper & Embedded \\
\hline $\begin{array}{l}\text { National FinSote } \\
\text { study (FinSote) }^{d}\end{array}$ & 1987 to date & Annual & 10,000 & $18+$ & $\begin{array}{l}\text { Self- } \\
\text { administered }\end{array}$ & $\begin{array}{l}\text { Pen and paper, } \\
\text { Computer or online } \\
\text { survey }\end{array}$ & Embedded \\
\hline \multicolumn{8}{|l|}{ Germany $^{a}$} \\
\hline $\begin{array}{l}\text { German Nutrition } \\
\text { Survey (NVS II) }\end{array}$ & $2005-2007$ & Random & 14,291 & $14-80$ & $\begin{array}{l}\text { Self- } \\
\text { administered }\end{array}$ & Pen and paper & Embedded \\
\hline $\begin{array}{l}\text { German Health } \\
\text { Interview and } \\
\text { Examination Survey } \\
\text { for Adults (DEGS) }\end{array}$ & $\begin{array}{l}\text { 2008-2011 (some } \\
\text { participated in } 98 \mathrm{GNHI} \\
\text { ES98) }\end{array}$ & Random & 7988 & $18-79$ & $\begin{array}{l}\text { Self- } \\
\text { administered }\end{array}$ & Pen and paper & Embedded \\
\hline \multicolumn{8}{|l|}{ Greece $^{a}$} \\
\hline $\begin{array}{l}\text { Sports for all } \\
\text { programs }(\text { SFAP) }\end{array}$ & 1983, 1995 to date & Annual & 60,000 & $5-80$ & $\begin{array}{l}\text { Self- } \\
\text { administered }\end{array}$ & Pen and paper & Embedded \\
\hline \multicolumn{8}{|l|}{ Ireland } \\
\hline $\begin{array}{l}\text { Irish Sports Monitor } \\
\text { (ISM) }\end{array}$ & $\begin{array}{l}\text { 2007, 2008, 2009, 2011, } \\
2013,2015,2017\end{array}$ & 2 years & 8500 & $16+$ & Interview & Telephone interview & $\begin{array}{l}\text { Exclusively } \\
\mathrm{PA} / \mathrm{SB} / \mathrm{SP}\end{array}$ \\
\hline $\begin{array}{l}\text { Healthy Ireland } \\
\text { Survey (HIS) }\end{array}$ & 2015-2018 to date & Annual & 7500 & $15+$ & Interview & $\begin{array}{l}\text { Computer or online } \\
\text { survey }\end{array}$ & Embedded \\
\hline The Irish Longitudinal & 2009 to date & Annual & 8000 & $50+$ & Interview & Face to face interview & Embedded \\
\hline
\end{tabular}


Table 1 Basic characteristics of movement behaviours monitoring systems across the European Union (Continued)

\begin{tabular}{|c|c|c|c|c|c|c|}
\hline \multirow{2}{*}{$\begin{array}{l}\text { Country, name of the } \\
\text { system and } \\
\text { (abbreviation) }\end{array}$} & \multirow[t]{2}{*}{ Years of monitoring } & \multirow{2}{*}{$\begin{array}{l}\text { Frequency } \\
\text { of } \\
\text { monitoring }\end{array}$} & \multirow{2}{*}{$\begin{array}{l}\text { App. } \\
\text { number of } \\
\text { participants }\end{array}$} & \multirow{2}{*}{$\begin{array}{l}\text { Age range } \\
\text { of } \\
\text { participants }\end{array}$} & Administration & \multirow{2}{*}{$\begin{array}{l}\text { Embedded } \\
\text { or } \\
\text { exclusively } \\
\text { PA/SB/SP }\end{array}$} \\
\hline & & & & & Mode & \\
\hline
\end{tabular}

\begin{tabular}{|c|c|c|c|c|c|c|c|}
\hline $\begin{array}{l}\text { Study on Ageing } \\
\text { (TILDA) }\end{array}$ & & & & & & & \\
\hline \multicolumn{8}{|l|}{ Italy ${ }^{a}$} \\
\hline $\begin{array}{l}\text { Aspetti della vita } \\
\text { quotidiana (AVQ) }\end{array}$ & 1993 to date & Annual & $50,000 /$ year & $18-64$ & $\begin{array}{l}\text { Self- } \\
\text { administered }\end{array}$ & Pen and paper & Embedded \\
\hline $\begin{array}{l}\text { Progressi Delle } \\
\text { Aziende Sanitarie per } \\
\text { la Salute in Italia } \\
\text { (PASSI) }\end{array}$ & 2010 to date & Annual & $35,000 /$ year & $18-69$ & Interview & Telephone interview & Embedded \\
\hline $\begin{array}{l}\text { Italian Population } \\
\text { Survey on Alcohol } \\
\text { and other Drugs (IPSA } \\
\text { D) }\end{array}$ & $2001-2009$ & 2 years & $85,000 /$ year & $15-64$ & $\begin{array}{l}\text { Self- } \\
\text { administered }\end{array}$ & Pen and paper & Embedded \\
\hline \multicolumn{8}{|l|}{ Lithuania } \\
\hline $\begin{array}{l}\text { Study of the physical } \\
\text { status of adults in } \\
\text { Lithuania (SPS) }\end{array}$ & 2010; 2014 & 5 years & 5000 & $18-75$ & Interview & Pen and paper & Embedded \\
\hline $\begin{array}{l}\text { National Survey on } \\
\text { Physical Activity in } \\
\text { Lithuania (SPA) }\end{array}$ & $\begin{array}{l}2002,2007,2011,2013- \\
2017\end{array}$ & Annual & 1525 & $15-75$ & Interview & $\begin{array}{l}\text { Computer aided } \\
\text { personal interview }\end{array}$ & $\begin{array}{l}\text { Exclusively } \\
\mathrm{PA} / \mathrm{SB} / \mathrm{SP}\end{array}$ \\
\hline \multicolumn{8}{|l|}{ The Netherlands } \\
\hline $\begin{array}{l}\text { Health Survey/ } \\
\text { Lifestyle Monitor (HS/ } \\
\text { LSM) }\end{array}$ & 1981 to date & Annual & 10,000 & $4+$ & $\begin{array}{l}\text { Self- } \\
\text { administered, } \\
\text { Interview, } \\
\text { Other }\end{array}$ & $\begin{array}{l}\text { Computer aided web } \\
\text { interview, computer- } \\
\text { assisted telephone } \\
\text { interview }\end{array}$ & Embedded \\
\hline $\begin{array}{l}\text { Additional module } \\
\text { Physical activity and } \\
\text { Accidents/Lifestyle } \\
\text { Monitor (LSM-A PA \& } \\
\text { accidents) }\end{array}$ & 2015 to date & 2 years & 10,000 & $4+$ & $\begin{array}{l}\text { Self- } \\
\text { administered, } \\
\text { interview, } \\
\text { Other }\end{array}$ & $\begin{array}{l}\text { Computer aided web } \\
\text { interview, Computer- } \\
\text { assisted telephone } \\
\text { interview, Computer } \\
\text { aided personal } \\
\text { interview }\end{array}$ & Embedded \\
\hline $\begin{array}{l}\text { Dutch National Food } \\
\text { Consumption Survey } \\
\text { (DNFCS) }\end{array}$ & $\begin{array}{l}2003,2005-2006,2007- \\
2010,2010-2012,2012- \\
2016\end{array}$ & $2-3$ & $\begin{array}{l}\text { depends on } \\
\text { year }\end{array}$ & $3+$ & $\begin{array}{l}\text { Self- } \\
\text { administered }\end{array}$ & $\begin{array}{l}\text { Pen and paper, } \\
\text { Computer or online } \\
\text { survey }\end{array}$ & Embedded \\
\hline $\begin{array}{l}\text { 'Omnibus of } \\
\text { recreation' (VTO) }\end{array}$ & 2012 to date & 2 years & 3000 & $6+$ & $\begin{array}{l}\text { Self- } \\
\text { administered, } \\
\text { interview, } \\
\text { Other }\end{array}$ & $\begin{array}{l}\text { Computer or online } \\
\text { survey, Computer aided } \\
\text { personal interview }\end{array}$ & Embedded \\
\hline \multicolumn{8}{|l|}{ Portugal } \\
\hline $\begin{array}{l}\text { National Physical } \\
\text { Activity and Sport } \\
\text { Monitoring System } \\
\text { (NPASMS) }\end{array}$ & $\begin{array}{l}2006-2009 \text { and 2017- } \\
2018\end{array}$ & 4 years & 1250 & $18-64$ & Interview & Pen and paper & Embedded \\
\hline \multicolumn{8}{|l|}{ Romania } \\
\hline $\begin{array}{l}\text { Study on behavioural } \\
\text { determinants of } \\
\text { health status for the } \\
\text { adult population in } \\
\text { Romania } \\
\text { (CompSanRO) }\end{array}$ & 2016 to date & 2 years & 1538 & $18-64$ & Interview & Telephone interview & Embedded \\
\hline \multicolumn{8}{|l|}{ Slovenia } \\
\hline $\begin{array}{l}\text { Slovenian Public } \\
\text { Opinion Survey } \\
\text { (SPOS) }\end{array}$ & $\begin{array}{l}\text { 1973, 1975-76, 1980, } \\
\text { 1983, 1986, 1989, 1992, } \\
\text { 1996, 1997, 1998, 1999, } \\
2000,2001,2004,2006\end{array}$ & Random & 1250 & $15+$ & Interview & Pen and paper & Embedded \\
\hline
\end{tabular}


Table 1 Basic characteristics of movement behaviours monitoring systems across the European Union (Continued)

\begin{tabular}{|c|c|c|c|c|c|c|c|}
\hline \multirow{2}{*}{$\begin{array}{l}\text { Country, name of the } \\
\text { system and } \\
\text { (abbreviation) }\end{array}$} & \multirow[t]{2}{*}{ Years of monitoring } & \multirow{2}{*}{$\begin{array}{l}\text { Frequency } \\
\text { of } \\
\text { monitoring }\end{array}$} & \multirow{2}{*}{$\begin{array}{l}\text { App. } \\
\text { number of } \\
\text { participants }\end{array}$} & \multirow{2}{*}{$\begin{array}{l}\text { Age range } \\
\text { of } \\
\text { participants }\end{array}$} & \multicolumn{2}{|c|}{ Administration } & \multirow{2}{*}{$\begin{array}{l}\text { Embedded } \\
\text { or } \\
\text { exclusively } \\
\text { PA/SB/SP }\end{array}$} \\
\hline & & & & & Mode & Means & \\
\hline & 2008,2012 & & & & & & \\
\hline $\begin{array}{l}\text { Collaboration for } \\
\text { Integrated } \\
\text { Noncommunicable } \\
\text { Disease Intervention } \\
\text { (CINDI) }\end{array}$ & 2001, 2004, 2008, 2012 & Random & 16,000 & $25-74$ & $\begin{array}{l}\text { Self- } \\
\text { administered }\end{array}$ & $\begin{array}{l}\text { Pen and paper, } \\
\text { Computer or online } \\
\text { survey }\end{array}$ & Embedded \\
\hline \multicolumn{8}{|l|}{ Spain $^{a}$} \\
\hline $\begin{array}{l}\text { Encuesta Nacional de } \\
\text { Salud Española } \\
\text { (Spanish National } \\
\text { Health Survey) (ENSE) }\end{array}$ & 1987-2017 & Random & 15,0260 & $15-85$ & Interview & Face to face interview & $\begin{array}{l}\text { Exclusively } \\
\mathrm{PA} / \mathrm{SB} / \mathrm{SP}\end{array}$ \\
\hline $\begin{array}{l}\text { Consejo Superior de } \\
\text { Deportes (CSD) }\end{array}$ & $2005-2015$ & 5 years & 12,000 & $15+$ & Interview & $\begin{array}{l}\text { Telephone interview, } \\
\text { Computer or online } \\
\text { survey }\end{array}$ & Embedded \\
\hline \multicolumn{8}{|l|}{ Sweden } \\
\hline $\begin{array}{l}\text { The national public } \\
\text { health survey Health } \\
\text { on equal terms (HLV) }\end{array}$ & 2004-2016, 2018 & 2 years & 40,000 & $16-84$ & $\begin{array}{l}\text { Self- } \\
\text { administered }\end{array}$ & $\begin{array}{l}\text { Pen and paper, } \\
\text { Computer or online } \\
\text { survey }\end{array}$ & Embedded \\
\hline
\end{tabular}

included systems that were not a part of multinational surveys (e.g., EHIS). The main results of this study are: 1) PA, SB and SP of adults are still not being systematically monitored in $11 / 27$ EU countries, while only 8 Member States are monitoring all three behaviours; 2) only the Netherlands, Portugal and Slovenia hold monitoring systems that encompass all three behaviours while covering most of the domains and dimensions of PA/ $\mathrm{SB} / \mathrm{SP}$; 3) measurement tools being used show large heterogeneity across the countries with established monitoring systems; and 4) existing monitoring systems typically rely on PA questionnaires, while device-based assessment of PA/SB is scarce.

Although international surveillance systems enable between-countries comparisons, they typically lack detail and fail to consider cultural context of a specific environment. To this end, national systems that consider specific cultural contexts are needed for optimal policymaking. In a recent comprehensive analysis performed by the WHO, almost all EU Member States have been identified as having at least one surveillance system for PA [16]. In contrast, by excluding EU-wide surveillance (e.g., EHIS), and by insisting on more stringent inclusion criteria for surveillance (i.e., by including only systematic collection, analysis and interpretation of the health-related data and not sporadic, individual research studies) this study found that only a little over half of EU countries systematically monitor at least one of the concepts related to PA. Some of these monitoring systems date back to the twentieth century, as early as 1964 (Denmark) and 1973 (Slovenia), but most of the countries started monitoring PA in the 2000s. The growth of PA monitoring systems in EU coincides with an increase in PA promotion and other health-enhancing initiatives and policies [3, 4, 6-8]. Nevertheless, 11 Member States are forced to rely exclusively on estimates coming from EU-wide surveillance systems in the policy creation and policy evaluation process. These systems do not provide any information on domains or dimensions of PA, they typically lack detailed estimates across regions, and they are run only every 5-6 years. Although EU-wide systems are vital for policy making at the EU level, all these limitations impede their use for timely and efficient policymaking at a national level. In conclusion, much work is ahead if systematic and detailed surveillance of PArelated behaviours on a national level is to be achieved across the whole EU.

Next, we found that more than one half of existing surveillance systems exclude individuals older than 80 years. Considering that more than 27 million people older than 80 were living in the EU in 2016 [18], and that the share of elderly people has been projected to continue increasing, it is of paramount importance to also include the oldest age groups in the surveillance of $\mathrm{PA} / \mathrm{SB} / \mathrm{SP}$. Besides the well-known benefits of PA for cardiovascular and metabolic health, in older adults PA 
Table 2 Domains and dimensions of movement behaviours covered by each monitoring system

\begin{tabular}{|c|c|c|c|c|c|c|c|}
\hline \multirow[t]{2}{*}{ Country, system } & \multirow[t]{2}{*}{ PAQ } & \multirow{2}{*}{$\begin{array}{l}\text { Physical activity } \\
\text { Type \& Domain }\end{array}$} & \multirow[b]{2}{*}{ Dimension } & \multicolumn{2}{|c|}{ Sedentary behaviour } & \multicolumn{2}{|c|}{ Sport participation } \\
\hline & & & & Domain & Dimension & Domain & Dimension \\
\hline \multicolumn{8}{|l|}{ Belgium } \\
\hline $\begin{array}{l}\text { Belgian National Food } \\
\text { Consumption Survey }\end{array}$ & $\begin{array}{l}\text { IPAQ-LF, } \\
\text { EHIS-PAQ }\end{array}$ & $\begin{array}{l}\text { Occupational, transport, } \\
\text { cycling/walking, MVPA }\end{array}$ & $\begin{array}{l}\text { Frequency, } \\
\text { duration, } \\
\text { intensity, } \\
\text { total } \\
\text { volume }\end{array}$ & - & - & Unspecified & $\begin{array}{l}\text { Frequency, } \\
\text { duration }\end{array}$ \\
\hline BHIS & $\begin{array}{l}\text { IPAQ-LF, } \\
\text { EHIS-PAQ }\end{array}$ & $\begin{array}{l}\text { Leisure-time, } \\
\text { occupational, cycling/ } \\
\text { walking, MVPA, }\end{array}$ & $\begin{array}{l}\text { Frequency, } \\
\text { duration, } \\
\text { intensity, } \\
\text { total } \\
\text { volume }\end{array}$ & - & - & - & - \\
\hline \multicolumn{8}{|l|}{ Denmark } \\
\hline $\begin{array}{l}\text { Den Nationale } \\
\text { Sundhedsprofil }\end{array}$ & $\begin{array}{l}\text { NPAQ } \\
\text { Marshall D }\end{array}$ & $\mathrm{MVPA}^{+}$ & Duration & $\begin{array}{l}\text { Leisure-time, } \\
\text { transport, } \\
\text { school }\end{array}$ & Total volume & - & - \\
\hline $\begin{array}{l}\text { Danskernes motions- } \\
\text { og sportsvaner }\end{array}$ & $\begin{array}{l}\text { Newly } \\
\text { developed/ } \\
\text { adapted }\end{array}$ & - & - & - & - & $\begin{array}{l}\text { Organised, non- } \\
\text { organised, } \\
\text { competitive }\end{array}$ & $\begin{array}{l}\text { Frequency, } \\
\text { duration, } \\
\text { type of } \\
\text { sport }\end{array}$ \\
\hline \multicolumn{8}{|l|}{ Estonia } \\
\hline $\begin{array}{l}\text { Health Behaviour } \\
\text { among Estonian Adult } \\
\text { Population }\end{array}$ & $\begin{array}{l}\text { Newly } \\
\text { developed/ } \\
\text { adapted }\end{array}$ & - & - & - & - & Unspecified & $\begin{array}{l}\text { Frequency, } \\
\text { duration }\end{array}$ \\
\hline $\begin{array}{l}\text { National Physical } \\
\text { Activity Survey among } \\
\text { Estonian Adult } \\
\text { Population }\end{array}$ & $\begin{array}{l}\text { Newly } \\
\text { developed/ } \\
\text { adapted }\end{array}$ & Leisure-time & $\begin{array}{l}\text { Frequency, } \\
\text { duration }\end{array}$ & - & - & Unspecified & $\begin{array}{l}\text { Frequency, } \\
\text { duration, } \\
\text { type of } \\
\text { sport }\end{array}$ \\
\hline \multicolumn{8}{|l|}{ Finland } \\
\hline $\begin{array}{l}\text { National FinHealth } \\
\text { Study }\end{array}$ & $\begin{array}{l}\text { Newly } \\
\text { developed/ } \\
\text { adapted }\end{array}$ & $\begin{array}{l}\text { Leisure-time, } \\
\text { occupational, transport }\end{array}$ & $\begin{array}{l}\text { Frequency, } \\
\text { duration }\end{array}$ & - & - & $\begin{array}{l}\text { Organised, non- } \\
\text { organised }\end{array}$ & Frequency \\
\hline $\begin{array}{l}\text { Terveys } 2011 \text { (Health } \\
\text { 2000-2011) }\end{array}$ & IPAQ-LF & $\begin{array}{l}\text { Occupational, transport, } \\
\text { home/household, } \\
\text { cycling/walking }\end{array}$ & $\begin{array}{l}\text { Frequency, } \\
\text { duration, } \\
\text { intensity, } \\
\text { total } \\
\text { volume }\end{array}$ & $\begin{array}{l}\text { Leisure-time, } \\
\text { occupational, } \\
\text { transport }\end{array}$ & $\begin{array}{l}\text { Screen time, } \\
\text { sitting, total } \\
\text { volume }\end{array}$ & - & - \\
\hline $\begin{array}{l}\text { Health Behaviour and } \\
\text { Health among the } \\
\text { Finnish Retirement- } \\
\text { Age Population }\end{array}$ & $\begin{array}{l}\text { Newly } \\
\text { developed/ } \\
\text { adapted }\end{array}$ & cycling/walking, MVPA & $\begin{array}{l}\text { Frequency, } \\
\text { duration, } \\
\text { intensity }\end{array}$ & - & - & - & - \\
\hline $\begin{array}{l}\text { National FinSote } \\
\text { study }^{d}\end{array}$ & $\begin{array}{l}\text { Newly } \\
\text { developed/ } \\
\text { adapted }\end{array}$ & $\begin{array}{l}\text { Leisure-time, } \\
\text { occupational, transport }^{c}\end{array}$ & $\begin{array}{l}\text { Frequency, } \\
\text { duration, } \\
\text { intensity, } \\
\text { total } \\
\text { volume }^{c}\end{array}$ & $\begin{array}{l}\text { Leisure-time, } \\
\text { occupational }^{c}\end{array}$ & Total volume ${ }^{c}$ & - & - \\
\hline \multicolumn{8}{|l|}{ Germany } \\
\hline $\begin{array}{l}\text { German Nutrition } \\
\text { Survey }\end{array}$ & $\begin{array}{l}\text { Newly } \\
\text { developed/ } \\
\text { adapted }\end{array}$ & $\begin{array}{l}\text { Transport, cycling/ } \\
\text { walking, home/ } \\
\text { household, gardening, } \\
\text { house work, house } \\
\text { maintenance }\end{array}$ & Duration & - & - & Unspecified & Other \\
\hline $\begin{array}{l}\text { German Health } \\
\text { Interview and } \\
\text { Examination Survey } \\
\text { for Adults }\end{array}$ & $\begin{array}{l}\text { Newly } \\
\text { developed/ } \\
\text { adapted }\end{array}$ & MVPA & $\begin{array}{l}\text { Frequency, } \\
\text { duration }\end{array}$ & - & - & Unspecified & Duration \\
\hline \multicolumn{8}{|l|}{ Greece } \\
\hline Sports for all & Newly & Leisure-time & Frequency, & - & - & Organised & Frequency \\
\hline
\end{tabular}


Table 2 Domains and dimensions of movement behaviours covered by each monitoring system (Continued)

\begin{tabular}{|c|c|c|c|c|c|c|c|}
\hline \multirow[t]{2}{*}{ Country, system } & \multirow[t]{2}{*}{ PAQ } & \multirow{2}{*}{$\begin{array}{l}\text { Physical activity } \\
\text { Type \& Domain }\end{array}$} & \multirow[b]{2}{*}{ Dimension } & \multicolumn{2}{|c|}{ Sedentary behaviour } & \multicolumn{2}{|l|}{ Sport participation } \\
\hline & & & & Domain & Dimension & Domain & $\overline{\text { Dimension }}$ \\
\hline programs & $\begin{array}{l}\text { developed/ } \\
\text { adapted }\end{array}$ & & duration & & & & \\
\hline \multicolumn{8}{|l|}{ Ireland } \\
\hline Irish Sports Monitor & ISM & $\begin{array}{l}\text { Leisure-time, transport, } \\
\text { cycling/walking }\end{array}$ & & - & - & $\begin{array}{l}\text { Organised, non- } \\
\text { organised, competi- } \\
\text { tive, unspecified, } \\
\text { coaching/ } \\
\text { volunteering }\end{array}$ & $\begin{array}{l}\text { Frequency, } \\
\text { duration, } \\
\text { type of } \\
\text { sport, }\end{array}$ \\
\hline Healthy Ireland Survey & HIS & $\begin{array}{l}\text { Leisure-time, transport, } \\
\text { cycling/walking }\end{array}$ & $\begin{array}{l}\text { Frequency, } \\
\text { total } \\
\text { volume }\end{array}$ & Total volume $e^{c}$ & Sitting & - & - \\
\hline $\begin{array}{l}\text { The Irish Longitudinal } \\
\text { Study on Ageing }\end{array}$ & IPAQ-SF & $\begin{array}{l}\text { Leisure-time, transport, } \\
\text { home/household, } \\
\text { cycling/walking, } \\
\text { coaching, volunteering }\end{array}$ & $\begin{array}{l}\text { Frequency, } \\
\text { duration, } \\
\text { intensity, } \\
\text { total } \\
\text { volume }\end{array}$ & - & - & - & - \\
\hline \multicolumn{8}{|l|}{ Italy } \\
\hline $\begin{array}{l}\text { Aspetti della vita } \\
\text { quotidiana }\end{array}$ & $\begin{array}{l}\text { Newly } \\
\text { developed/ } \\
\text { adapted }\end{array}$ & PA & Frequency & - & - & Unspecified & $\begin{array}{l}\text { Frequency, } \\
\text { duration }\end{array}$ \\
\hline $\begin{array}{l}\text { Progressi Delle } \\
\text { Aziende Sanitarie per } \\
\text { la Salute in Italia }\end{array}$ & PASSI & $\begin{array}{l}\text { PA at work and out of } \\
\text { work }\end{array}$ & $\begin{array}{l}\text { Frequency, } \\
\text { duration, } \\
\text { intensity }\end{array}$ & - & - & - & - \\
\hline $\begin{array}{l}\text { Italian Population } \\
\text { Survey on Alcohol } \\
\text { and other Drugs }\end{array}$ & IPSAD & PA & $\begin{array}{l}\text { Frequency, } \\
\text { duration, } \\
\text { intensity }\end{array}$ & - & - & - & - \\
\hline \multicolumn{8}{|l|}{ Lithuania } \\
\hline $\begin{array}{l}\text { Study of the physical } \\
\text { status of adults in } \\
\text { Lithuania }\end{array}$ & $\begin{array}{l}\text { Adapted } \\
\text { IPAQ-SF }\end{array}$ & $\begin{array}{l}\text { Leisure-time, } \\
\text { occupational, transport }\end{array}$ & $\begin{array}{l}\text { Total } \\
\text { volume }\end{array}$ & $\begin{array}{l}\text { Leisure-time, } \\
\text { occupational, } \\
\text { transport }\end{array}$ & Total volume & - & - \\
\hline $\begin{array}{l}\text { National Survey on } \\
\text { Physical Activity in } \\
\text { Lithuania }\end{array}$ & GPAQ & $\begin{array}{l}\text { Leisure-time, } \\
\text { occupational, transport, } \\
\text { home/household, } \\
\text { cycling/walking }\end{array}$ & Frequency & - & - & - & - \\
\hline \multicolumn{8}{|l|}{ the Netherlands } \\
\hline $\begin{array}{l}\text { Health Survey/Lifestyle } \\
\text { Monitor }\end{array}$ & SQUASH & $\begin{array}{l}\text { Leisure-time, } \\
\text { occupational, transport, } \\
\text { home/household, } \\
\text { cycling/walking }\end{array}$ & $\begin{array}{l}\text { Frequency, } \\
\text { duration, } \\
\text { intensity, } \\
\text { total } \\
\text { volume }^{c}\end{array}$ & - & - & $\begin{array}{l}\text { Organised, non- } \\
\text { organised, } \\
\text { competitive }\end{array}$ & $\begin{array}{l}\text { Frequency, } \\
\text { duration, } \\
\text { type of } \\
\text { sport, other }\end{array}$ \\
\hline $\begin{array}{l}\text { Additional module } \\
\text { Physical activity and } \\
\text { Accidents/Lifestyle } \\
\text { Monitor }\end{array}$ & $\begin{array}{l}\text { SQUASH } \\
\text { Marshall D }\end{array}$ & $\begin{array}{l}\text { Leisure-time, } \\
\text { occupational, transport, } \\
\text { home/household, } \\
\text { cycling/walking }\end{array}$ & $\begin{array}{l}\text { Frequency, } \\
\text { duration, } \\
\text { intensity, } \\
\text { total } \\
\text { volume }^{c}\end{array}$ & $\begin{array}{l}\text { Leisure-time, } \\
\text { occupational, } \\
\text { transport, } \\
\text { school }\end{array}$ & $\begin{array}{l}\text { Screen time, } \\
\text { sitting, } \\
\text { reclining/lying } \\
\text { down, total } \\
\text { volume }\end{array}$ & $\begin{array}{l}\text { Organised, non- } \\
\text { organised, } \\
\text { competitive }\end{array}$ & $\begin{array}{l}\text { Frequency, } \\
\text { duration, } \\
\text { type of } \\
\text { sport, other }\end{array}$ \\
\hline $\begin{array}{l}\text { Dutch National Food } \\
\text { Consumption Survey }\end{array}$ & SQUASH & $\begin{array}{l}\text { Leisure-time, } \\
\text { occupational, transport, } \\
\text { home/household, } \\
\text { cycling/walking, sport, } \\
\text { gardening }\end{array}$ & Duration & - & - & - & - \\
\hline $\begin{array}{l}\text { 'Omnibus of } \\
\text { recreation' }\end{array}$ & $\mathrm{RSO}$ & - & - & - & - & $\begin{array}{l}\text { Organised, non- } \\
\text { organised, } \\
\text { competitivet }\end{array}$ & $\begin{array}{l}\text { Frequency, } \\
\text { type of } \\
\text { sport }\end{array}$ \\
\hline \multicolumn{8}{|l|}{ Portugal } \\
\hline $\begin{array}{l}\text { National Physical } \\
\text { Activity and Sport } \\
\text { Monitoring System }\end{array}$ & IPAQ-SF & $\begin{array}{l}\text { Leisure-time, } \\
\text { occupational, transport, } \\
\text { home/household, }\end{array}$ & $\begin{array}{l}\text { Frequency, } \\
\text { duration, } \\
\text { intensity, }\end{array}$ & $\begin{array}{l}\text { Leisure-time, } \\
\text { occupational, } \\
\text { transport }\end{array}$ & $\begin{array}{l}\text { Screen time, } \\
\text { sitting, } \\
\text { reclining/lying }\end{array}$ & $\begin{array}{l}\text { Organised, non- } \\
\text { organised }\end{array}$ & $\begin{array}{l}\text { Frequency, } \\
\text { duration, } \\
\text { type of }\end{array}$ \\
\hline
\end{tabular}


Table 2 Domains and dimensions of movement behaviours covered by each monitoring system (Continued)

\begin{tabular}{|c|c|c|c|c|c|c|c|}
\hline \multirow[t]{2}{*}{ Country, system } & \multirow[t]{2}{*}{ PAQ } & \multirow{2}{*}{$\begin{array}{l}\text { Physical activity } \\
\text { Type \& Domain }\end{array}$} & \multirow[b]{2}{*}{ Dimension } & \multicolumn{2}{|c|}{ Sedentary behaviour } & \multicolumn{2}{|c|}{ Sport participation } \\
\hline & & & & Domain & Dimension & Domain & Dimension \\
\hline & & cycling/walking & $\begin{array}{l}\text { total } \\
\text { volume }\end{array}$ & & $\begin{array}{l}\text { down, total } \\
\text { volume }\end{array}$ & & sport \\
\hline \multicolumn{8}{|l|}{ Romania } \\
\hline $\begin{array}{l}\text { Study on behavioural } \\
\text { determinants of } \\
\text { health status for the } \\
\text { adult population in } \\
\text { Romania }\end{array}$ & $\begin{array}{l}\text { National } \\
\text { institute of } \\
\text { public health } \\
\text { questionnaire }\end{array}$ & $\begin{array}{l}\text { Leisure-time, } \\
\text { occupational, transport, } \\
\text { cycling/walking }\end{array}$ & $\begin{array}{l}\text { Frequency, } \\
\text { duration, } \\
\text { intensity }\end{array}$ & $\begin{array}{l}\text { Leisure-time, } \\
\text { occupational, } \\
\text { transport }\end{array}$ & Total volume & Unspecified & Duration \\
\hline \multicolumn{8}{|l|}{ Slovenia } \\
\hline $\begin{array}{l}\text { Slovenian Public } \\
\text { Opinion Survey }\end{array}$ & $\begin{array}{l}\text { The Sport- } \\
\text { recreational } \\
\text { activity of } \\
\text { Slovenian }\end{array}$ & - & - & - & - & $\begin{array}{l}\text { Organised, non- } \\
\text { organised, } \\
\text { competitive }\end{array}$ & $\begin{array}{l}\text { Frequency, } \\
\text { duration, } \\
\text { type of } \\
\text { sport }\end{array}$ \\
\hline $\begin{array}{l}\text { Collaboration for } \\
\text { Integrated } \\
\text { Noncommunicable } \\
\text { Disease Intervention }\end{array}$ & $\begin{array}{l}\text { Adapted } \\
\text { IPAQ-SF }\end{array}$ & $\begin{array}{l}\text { Leisure-time, } \\
\text { occupational, transport, } \\
\text { home/household, } \\
\text { cycling/walking }\end{array}$ & $\begin{array}{l}\text { Frequency, } \\
\text { duration, } \\
\text { intensity }\end{array}$ & $\begin{array}{l}\text { Leisure-time, } \\
\text { occupational, } \\
\text { transport }\end{array}$ & $\begin{array}{l}\text { Screen time, } \\
\text { sitting }\end{array}$ & - & - \\
\hline \multicolumn{8}{|l|}{ Spain } \\
\hline $\begin{array}{l}\text { Encuesta Nacional de } \\
\text { Salud Española }\end{array}$ & IPAQ - SF & $\begin{array}{l}\text { Leisure-time, } \\
\text { occupational }\end{array}$ & Frequency & $\begin{array}{l}\text { Leisure-time, } \\
\text { occupational }\end{array}$ & Sitting & - & - \\
\hline $\begin{array}{l}\text { Consejo Superior de } \\
\text { Deportes }\end{array}$ & IPAQ - SF & Leisure-time & $\begin{array}{l}\text { Frequency, } \\
\text { duration }\end{array}$ & & & $\begin{array}{l}\text { Organised, non- } \\
\text { organised, } \\
\text { competitive }\end{array}$ & $\begin{array}{l}\text { Frequency, } \\
\text { duration, } \\
\text { type of } \\
\text { sport }\end{array}$ \\
\hline \multicolumn{8}{|l|}{ Sweden } \\
\hline $\begin{array}{l}\text { The national public } \\
\text { health survey Health } \\
\text { on equal terms }\end{array}$ & IPAQ - SF & MVPA & $\begin{array}{l}\text { Duration, } \\
\text { intensity }\end{array}$ & Total volume & Sitting & - & - \\
\hline
\end{tabular}

Legend.; ${ }^{\mathrm{c}}=$ depends on the year; ${ }^{\mathrm{d}}=$ FinSote study started as Health Behaviour and Health among the Finnish Adult Population and then The regional health and well-being survey; MVPA moderate and vigorous physical activity; GPAQ Global Physical Activity Questionnaire; NPAQ Nordic Physical Activity Questionnaire; ISMSF Indagine Statistica multiscopo sulle famiglie (Multi-purpose statistical survey on families); Marshall D Marshall Sitting Questionnaire; SQUASH Short Questionnaire to Assess Health enhancing physical activity; RSO Richtlijn voor Sportdeelname Onderzoek (Guideline for Sport participation Research)

has a vital role in the prevention of falls and falls-related injuries as well as in declines in bone and cognitive health.

Surveillance systems identified through this study typically included several domains of PA, most commonly leisure time and transport domains. At the same time, these two domains are the ones most amenable to interventions. With regard to the dimensions of PA, duration and frequency are being reported far more often than intensity. Although intensity of PA is the dimension that is the most difficult to assess, its importance has been repeatedly highlighted [19-21]. Unfortunately, even though the information about PA of different intensity is obviously available (e.g., from the IPAQ questionnaire), many surveillance systems fail to analyse and report these data. SB is monitored less frequently than PA, despite recent research showing the downsides of high $\mathrm{SB}$ and its deleterious effects on health [22-24]. Even when assessed, SB is typically conceptualised through only one dimension, mostly sitting, without any effort made to distinguish the context (e.g., watching TV, reading, playing, etc.). Given the accumulating evidence that unique sedentary behaviours relate to different health outcomes, including a number of SB dimensions should be encouraged. Next, in line with the recently introduced paradigm of 24-h movement guidelines [25], optimal surveillance should strive to include both PA and $\mathrm{SB}$, while also assessing sleep. As global or EU-wide health surveillance systems (e.g., The Global Health Observatory, The European Health Interview Survey) do not include information on sleep duration, and since we did not collect data on sleep for national surveillance systems included here, it was not possible to identify countries in the EU that monitor all movement behaviours and are therefore able to assess compliance with the 24-h movement guidelines. Still, as we identified only 10 Member States that are monitoring both PA and $\mathrm{SB}$, such estimates are evidently still not possible in the larger part of the EU.

Even though it has been well established that devicemeasured PA and SB are more reliable and valid compared to self-report [26-28], nearly all $16 \mathrm{EU}$ countries 


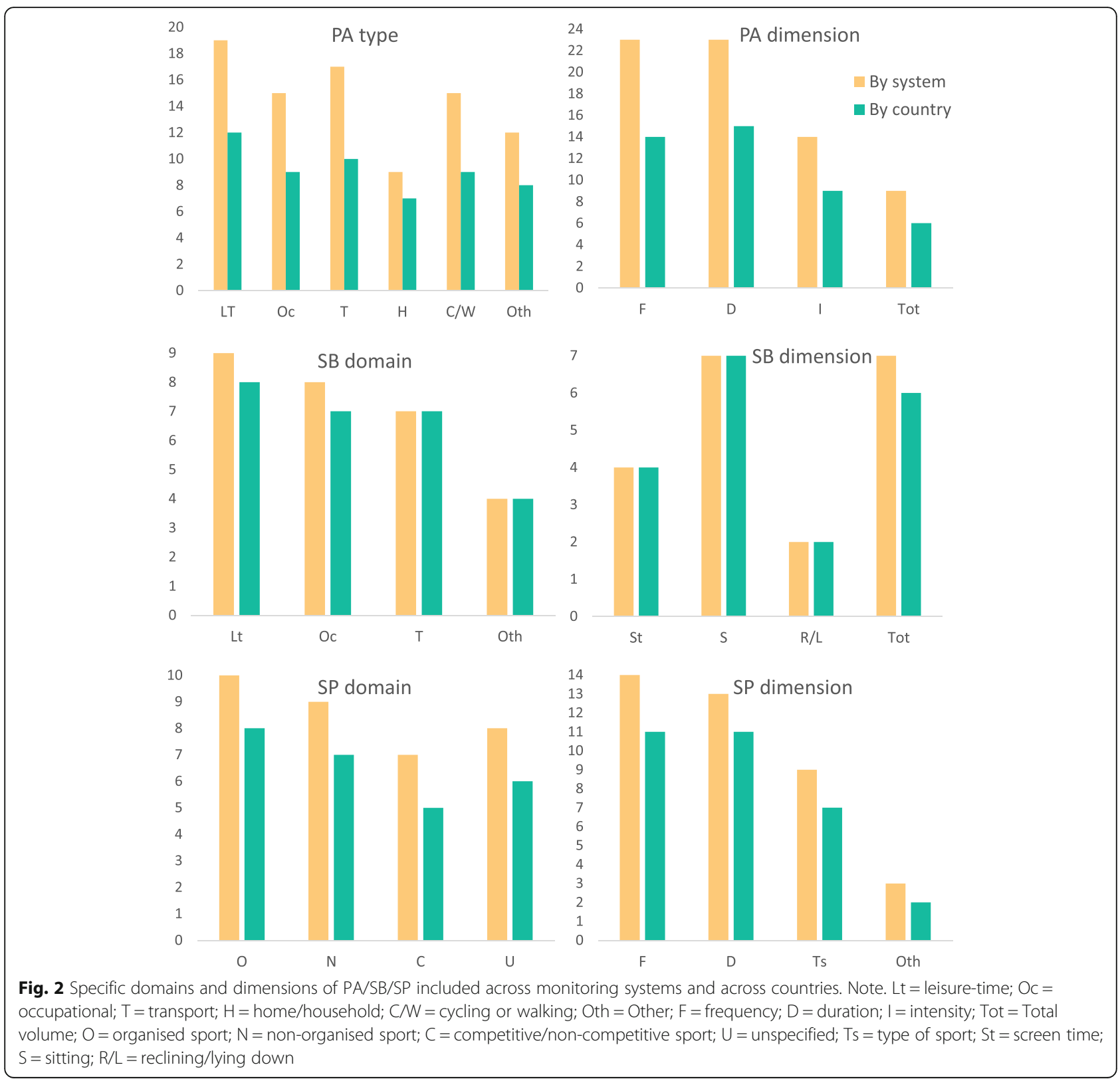

with established national surveillance systems are relying exclusively on self-report for monitoring PA and SB, probably because they are economically and logistically more suitable for large surveys. Only three countries have reported monitoring PA with accelerometers (Finland, Ireland and Portugal). Yet, these assessments are not being performed on a regular basis and include a rather small number of participants. At the same time, several population-based research studies that had been set-up in the last few years and assessed PA via accelerometers in >100,000 individuals, have shown that device-measured PA is feasible even on a large scale (e.g., UK Biobank study). Of note, the availability of device-based measures does not mean that the use of self-report methods should be discontinued. On the contrary, device-based assessment methods for PA/SB need to be accompanied with questionnaires that cover a wide range of domains and contexts of these behaviours in order to ensure the detail and the granularity of the data needed for data-informed policy making [29].

Surprisingly, many countries that have opted to perform cross-cultural adaptation of the existing questionnaires, have failed to confirm their measurement properties after the process. The fact that most countries are using questionnaires of unknown validity and reliability undermines the trustworthiness of conclusions based on the collected data and hampers the evaluation of the effectiveness of related policy measures. 
Our study has several strengths. This is the first study to offer a very extensive overview of national surveillance systems for several movement-related behaviours across EU. First, we collected detailed data from all EU countries. Second, we extended our focus from PA to include other behaviours related to energy expenditure such as SB and SP. Finally, we analysed details such as domains and dimensions for all three behaviours.

Still, this study is not without limitations. First, despite several attempts we have not been able to contact seven countries via an online questionnaire (Belgium, Denmark, Finland, Germany, Greece, Slovakia and Spain), so the data had to be extracted from earlier studies or received through contact with independent national experts. As a result, some information for these 7 Member States might be incomplete or outdated. Second, we asked for details on the dimensions and domains of PA, but did not gather data on the type of PA. Hence, it was not possible to determine the extent to which national surveillance systems are able to evaluate muscle strengthening and balance elements of PA recommendations. Third, although we collected very comprehensive data on systems that monitor three distinct behaviours (i.e., $\mathrm{PA} / \mathrm{SB} / \mathrm{SP}$ ), we did not request information on sleep surveillance. Consequently, we were not able to identify countries in the EU that are capable of evaluating adherence to the 24-h movement guidelines.

\section{Conclusion}

Surveillance of PA is still not systematic in almost half EU Member States, and SB or SP are being monitored even less frequently. Besides, existing surveillance systems typically fail to assess all dimensions and domains of PA/SB/SP, and only three countries (the Netherlands, Portugal and Slovenia) run monitoring systems that encompass all three behaviours while covering most of the domains and dimensions of PA/SB/SP. Hence, additional efforts in advocacy of PA surveillance are needed. In order to facilitate the assessment of existing policies and optimise the effectiveness of new policies, countries should be encouraged to implement regular monitoring of PA with valid instruments for PA assessment. Moreover, embedding $\mathrm{SB}$ or 24-h movement behaviours assessment, together with SP, in existing and new surveillance systems should be advocated. The addition of device-based assessment methods for PA/SB to questionnaires that cover a wide range of domains and contexts of these behaviours would ensure the detail and the quality of the data needed for data-informed policy making. Finally, we are witnessing unprecedented restrictions in PA being implemented on a population level as a response to the current public-health crisis related to the COVID-19 pandemic. At the same time, work dynamics have transformed with the increase in remote work. As this is projected to reshape work environments in the long-term, monitoring occupational PA and SB will become more important. Comprehensive, continuous surveillance of all movement-related behaviours could have a vital role in creating and evaluating policies designed to combat the inactivity pandemics in the post COVID era.

\section{Abbreviations \\ DEDIPAC: Determinants of diet and physical activity; EHIS: European Health Interview Survey; EHIS-PAQ: European Health Interview Survey Physical activity questionnaire; EU: European Union; EUPASMOS: European Physical Activity and Sports Monitoring System; HEPA: Health-enhancing physical activity; IPAQ: International Physical Activity Questionnaire; PA: Physical activity; PAQ: Physical activity questionnaire; SB: Sedentary behavior; SP: Sport participation; SQUASH: Short Questionnaire to Assess Health enhancing physical activityWHO - World Health Organization}

\section{Acknowledgements}

The authors also acknowledge the support of Stephen Whiting and Lea Nash Castro affiliated with the World Health Organisation at the time of the study, and the help of the EU HEPA focal points and other national representatives who provided information on their national PA/SB/SP monitoring systems: Austria: Susanne Ring-Dimitriou, University of Salzburg; Belgium: Greet Cardon, University of Gent, Bulgaria: Denitsa Pancheva, Ministry of Youth and Sports of Bulgaria; Croatia: Maroje Sorić, University of Zagreb; Cyprus: Michalis Michaelides, Cyprus sport organisation; Czech Republic: Hana Dvorakova, National Institute of Public Health; Denmark: Thomas Skovgaard, University of Southern Denmark and Tue Kristensen, Danish Health Authority; Estonia: Jarko Koort, Ministry of Culture; Finland: Tuija Tammelin, LIKES; France: David Thivel and Martine Duclos, Université Clermont Auvergne-CHU Clermont-Ferrand; Germany: Kristin Manz, Robert KochInstitut, and Thorsten Heuer, Max Rubner-Institut; Greece: Eleftheria Axioti, General Secretariat of Sports; Hungary: Réka Veress, Ministry of Human Capacities; Ireland: James Lavelle, Department of Transport, Tourism \& Sport; Italy: Laura Capranica, University of Rome Foro Italico; Latvia: Andra Fernate, Latvian Academy of Sport Education; Lithuania: Inga Gerulskienė, Ministry of Health; Luxembourg: Claude Scheuer, University of Luxembourg; Malta: Andrew Decelis, University of Malta; the Netherlands: Wanda Wendel-Vos and Ellen de Hollander, National Institute for Public Health and the Environment; Poland: Marian Stelmach, Pope John Paul II State School of Higher Education in Biala Podlaska; Portugal: Paulo Rocha, Portuguese Institute for Sport and Youth; Romania: Radu Bidiugan, National Research Institute for Sports;

Slovakia: Branislav Antala, Comenius University in Bratislava; Slovenia: Gregor Jurak, University of Ljubljana; Spain: Susana Aznar Lain, UCLM; Sweden: Marita Friberg, The Public Health Agency of Sweden.

\section{Authors' contributions}

Conceptualization, M.S. and G.J.; methodology, M.S., G.J., K.M., W.W.V., E.H..; formal analysis, K.M.; investigation, G.J., K.M. and M. S.; resources, G.J.; data curation, K.M.; writing —original draft preparation, K.M. and M.S.;

writing-review and editing, M.S., G.J., P.R., W.W.V., \& E.H.; visualization, K.M.; supervision, G.J. \& M.S.; project administration, G.J.; funding acquisition, P.R. \&G.J. All authors have read and agreed to the published version of the manuscript.

\section{Funding}

This study was part of the project EUPASMOS which was co-funded by the Erasmus+ Programme of the European Union No 590662-EPP-1-2017-1-PTSPO-SCP and Slovenian Research Agency.

\section{Availability of data and materials}

All data generated or analysed during this study are included in this published article. 


\section{Declarations}

\section{Ethics approval and consent to participate}

All methods and procedures of this study were in accordance with the Declaration of Helsinki and were approved by the Ethics Committee of the Faculty of Sport at the University of Ljubljana (ID: 10/2018). Having fully informed the participants about the aims and procedures of the study, their verbal consent was obtained. As only administrative data were recorded, and no personal data were collected, written informed consent was not required.

\section{Consent for publication}

Not applicable.

\section{Competing interests}

The authors declare that they have no competing interests.

\section{Author details}

${ }^{1}$ Faculty of Sport, University of Ljubljana, Gortanova 22, 1000 Ljubljana, Slovenia. ${ }^{2}$ Faculty of Kinesiology, University of Zagreb, Zagreb, Croatia. ${ }^{3}$ Portuguese Institute of Sport and Youth, Lisbon, Portugal. ${ }^{4}$ National Institute for Public Health and the Environment (RIVM), Bilthoven, Netherlands.

Received: 26 April 2021 Accepted: 22 September 2021

Published online: 07 October 2021

\section{References}

1. Bull FC, Al-Ansari SS, Biddle S, Borodulin K, Buman MP, Cardon G, et al. World Health Organization 2020 guidelines on physical activity and sedentary behaviour. Br J Sports Med. 2020;54(24):1451-62. https://doi.org/1 0.1136/bjsports-2020-102955.

2. Ding D, Lawson KD, Kolbe-Alexander TL, Finkelstein EA, Katzmarzyk PT, van Mechelen W, et al. The economic burden of physical inactivity: a global analysis of major non-communicable diseases. Lancet. 2016;388(10051): 1311-24. https://doi.org/10.1016/S0140-6736(16)30383-X.

3. World Health Organization. European food and nutrition action plan 20152020. Copenhagen: WHO Regional Committee for Europe; 2014.

4. World Health Organization. Physical activity strategy for the WHO European region 2016-2025. Copenhagen: WHO Regional Office for Europe; 2016. 32 p.

5. Council. Council Recommendation of 26 November 2013 on promoting health-enhancing physical activity across sectors. Off J Eur Union. 2013; 354(1):1-5.

6. European Commission. A growing health challenge for the EU. In: EU Action Plan on Childhood Obesity 2014-2020; 2014. p. 2-8.

7. World Health Organization. Global action plan on physical activity 20182030: more active people for a healthier world [Internet]. Geneva: World Health Organization: World Health Organization; 2018. p. 1-104. Available from: http://www.who.int/dietphysicalactivity/publications/9789241599979/ en/,\%0A http://www.who.int/news-room/detail/04-06-2018-who-launchesglobal-action-plan-on-physical-activity

8. World Health Organization. Global action plan for the prevention and control of noncommunicable diseases 2013-2020. Geneva: World Health Organization; 2013. p. 103.

9. European Commission. Report from the commission to the European parliament, the council, the European economic and social committee and the committee of the regions. Brussels; 2016.

10. Finger JD, Tafforeau J, Gisle L, Oja L, Ziese T, Thelen J, et al. Development of the European health interview survey-physical activity questionnaire (EHIS$\mathrm{PAQ}$ ) to monitor physical activity in the European Union. Arch Public Heal. 2015;73(1):59. https://doi.org/10.1186/s13690-015-0110-z.

11. European Parliament. Regulation (EC) no 1338/2008 of the European parliament and of the council of 16 December 2008 on community statistics on public health and health and safety at work. Off J Eur Union. 2008;12.

12. Eurostat European Commission. European Health Interview Survey (EHIS wave 3) Methodological manual [Internet]. Publications Office of the European Union. Available online at: http://www.google.rs/url. Luxembourg: Publications Office of the European Union; 2018. 222 p. Available from: https://ec.europa.eu/eurostat/documents/3859598/8762193/KS-02-18-240EN-N.pdf/5fa53ed4-4367-41c4-b3f5-260ced9ff2f6
13. Baumeister SE, Ricci C, Kohler S, Fischer B, Töpfer C, Finger JD, et al. Physical activity surveillance in the European Union: reliability and validity of the European health interview survey-physical activity questionnaire (EHIS-PAQ). Int J Behav Nutr Phys Act. 2016;13(1):61. https://doi.org/10.1186/s12966-0160386-6.

14. Sember V, Meh K, Sorić M, Starc G, Rocha P, Jurak G. Validity and reliability of international physical activity questionnaires for adults across EU countries: systematic review and Meta analysis. Int J Environ Res Public Health. 2020;17(19):7161. https://doi.org/10.3390/ijerph17197161.

15. World Health Organization. Factsheets on health-enhancing physical activity in the 28 EU Member States of the WHO European Region. Copenhagen: World Health Organization, Regional Office for Europe; 2015.

16. WHO/Europe. Physical activity country factsheets [Internet]. 2018 [cited 2021 Feb 24]. Available from: https://www.euro.who.int/en/health-topics/diseaseprevention/physical-activity/data-and-statistics/physical-activity-fact-sheets/ physical-activity-country-factsheets

17. Bel-Serrat S, Huybrechts I, Thumann BF, Hebestreit A, Abuja PM, De Henauw $\mathrm{S}$, et al. Inventory of surveillance systems assessing dietary, physical activity and sedentary behaviours in Europe: a DEDIPAC study. Eur J Pub Health. 2017;27(4):747-55. https://doi.org/10.1093/eurpub/ckx023.

18. Bourgeais V, Gereöffy A. Nearly 27 million people aged 80 or over in the European Union. Eurostat Newsrelease [Internet]. 2016 Sep 29;6. Available from: https://ec.europa.eu/eurostat/documents/2995521/7672228/3-2909201 6-AP-EN.pdf/4b90f6bb-43c1-45ed-985b-dfbe9564157a

19. Laukkanen JA, Rauramaa R, Mäkikallio TH, Toriola AT, Kurl S. Intensity of leisure-time physical activity and cancer mortality in men. Br I Sports Med. 2011;45(2):125-9. https://doi.org/10.1136/bjsm.2008.056713.

20. Amagasa S, Machida M, Fukushima N, Kikuchi H, Takamiya T, Odagiri Y, et al. Is objectively measured light-intensity physical activity associated with health outcomes after adjustment for moderate-to-vigorous physical activity in adults? A systematic review. Int J Behav Nutr Phys Act. 2018;15(1):1-13. https://doi.org/10.1186/s12966-018-0695-z.

21. Strain T, Wijndaele K, Dempsey PC, Sharp SJ, Pearce M, Jeon J, et al. Wearable-device-measured physical activity and future health risk. Nat Med. 2020;26(9):1385-91. https://doi.org/10.1038/s41591-020-1012-3.

22. Ekelund U, Steene-Johannessen J, Brown WJ, Fagerland MW, Owen N, Powell KE, et al. Does physical activity attenuate, or even eliminate, the detrimental association of sitting time with mortality? A harmonised metaanalysis of data from more than 1 million men and women. Lancet. 2016; 388(10051):1302-10. https://doi.org/10.1016/S0140-6736(16)30370-1.

23. Madhav KC, Sherchand SP, Sherchan S. Association between screen time and depression among US adults. Prev Med reports. 2017;8:67-71. https:// doi.org/10.1016/j.pmedr.2017.08.005.

24. Banks E, Jorm L, Rogers K, Clements M, Bauman A. Screen-time, obesity, ageing and disability: findings from 91266 participants in the 45 and up study. Public Health Nutr. 2011;14(1):34-43. https://doi.org/10.1017/S13 68980010000674.

25. Tremblay MS, Ross R. How should we move for health? The case for the 24hour movement paradigm. CMAJ. 2020;192(49):1728-9. https://doi.org/10.1 503/cmaj.202345.

26. Skender S, Ose J, Chang-Claude J, Paskow M, Brühmann B, Siegel EM, et al. Accelerometry and physical activity questionnaires-a systematic review. BMC Public Health. 2016;16(1):515. https://doi.org/10.1186/s12889-016-3172-0.

27. Prince SA, Adamo KB, Hamel ME, Hardt J, Gorber SC, Tremblay M. A comparison of direct versus self-report measures for assessing physical activity in adults: a systematic review. Int J Behav Nutr Phys Act. 2008;5(1): 56. https://doi.org/10.1186/1479-5868-5-56.

28. Westerterp KR. Assessment of physical activity: a critical appraisal. Eur J Appl Physiol. 2009;105(6):823-8. https://doi.org/10.1007/s00421-009-1000-2.

29. Sattler MC, Ainsworth BE, Andersen LB, Foster C, Hagströmer M, Jaunig J, et al. Physical activity self-reports: past or future? Br J Sports Med. 2021; 55(16):889-90.

\section{Publisher's Note}

Springer Nature remains neutral with regard to jurisdictional claims in published maps and institutional affiliations. 